to my students who are considering graduate studies and possible careers in research, as well as to friends and colleagues who are looking for a starting point to explore and understand the dynamics of natural and technological networks.

J. J. Collins is in the Center for BioDynamics and the Department of Biomedical Engineering, Boston University, Boston, Massachusetts 02215, USA.

\section{$\cdots \cdots \cdots \cdots \bullet$}

\section{All in the mind?}

\section{Placebo: The Belief Effect \\ by Dylan Evans}

HarperCollins: 2003. 240 pp. $£ 16.99$

\section{Walter A. Brown}

In recent years the placebo response has captivated both the scientific community and the general public. The Lancet ran a series of articles on the placebo effect in 1994, and the US National Institutes of Health (NIH) sponsored a conference on it in 2000 . Over the past five years, television programmes, newspapers and news magazines from around the world have carried stories about the placebo effect. Why the recent flurry of interest in a phenomenon that has been recognized for most of the past century and subjected to a low but steady rate of enquiry for 50 years?

Part of the fascination comes from the recent attention given to the mind-body relationship. Technology-driven advances in our knowledge of the brain and an interest in alternative and complementary medicine, with its emphasis on self-healing and spirituality, both focus attention on the links between mind, brain and illness, the arena in which placebo operates. The high visibility of clinical trials in recent years has also drawn attention to the placebo response. These trials keep telling us that for some conditions, such as depression and pain, placebo treatment brings almost as much benefit as 'real' drugs and surgical procedures. They have also spotlighted the ethics of placebo controls.

The placebo effect has long been considered a nuisance that obscured the identification of new treatments. For most of the past century, in the rare instances when doctors deliberately gave patients placebos, they did so to placate patients who were thought to be complaining too much, or to 'prove' that a symptom was psychologically based rather than 'real'.

But these views are changing. The placebo response is now widely regarded as a phenomenon worthy of study in its own right. After its placebo conference, for example, the NIH requested grant proposals to study both the basic mechanisms and the clinical application of the placebo response. Recent studies have shown that functional changes in the brain are associated with the placebo response in both depression and Parkinson's disease. And some clinicians are beginning to consider the placebo response as an integral part of healing, and are seeking ways to harness and enhance it.

Given the subtitle of Dylan Evans' book, one might expect it to offer a comprehensive assessment of the role of belief in the placebo effect. But that's not what we get. Instead there is a smorgasbord of facts and speculation presented in support of the author's main - and in my view thoroughly implausible — hypothesis that "all placeboresponsive conditions" involve activation of the inflammatory response and that placebos work by suppressing inflammation. If a highly placebo-responsive condition such as depression doesn't seem to involve the inflammatory response, Evans is undeterred: he comes up with a bit of information (in this instance one study showing that depressed patients have increased interleukin levels ) to convince us otherwise. He manages to fit Parkinson's disease into his model, along with a raft of other ills that few others would ever consider as diseases of inflammation. Evans does state at the outset that this is an unsubstantiated hypothesis, but later in the book, as he gets rolling, he seems to assume that he has demonstrated its validity.

His case is not helped by inaccuracies ranging from trivial errors of fact (he tells us that no study of depressed patients has included both a placebo and a no-treatment group, but there is at least one such study, and it is cited in a paper he reviews in depth), to misinformation (he says that placebos don't help asthma) and major misconceptions (that classical conditioning entails belief in the connection between the conditioned and unconditioned stimuli).

Evans does provide a reasonable critique of the widely discussed meta-analysis by Asbjørn Hróbjartsson and Peter Gøtzsche (New England Journal of Medicine 344, $1594-1602$; 2001) that compared placebo to no treatment. The data were largely misinterpreted, including by the authors, as showing that a placebo offers no more benefit than the passage of time. And Evans includes an entertaining chapter speculating on the evolution of the placebo response and how the capacity for it might be adaptive. It's utter nonsense, but I'm a natural-selection junkie and I enjoyed the attempt.

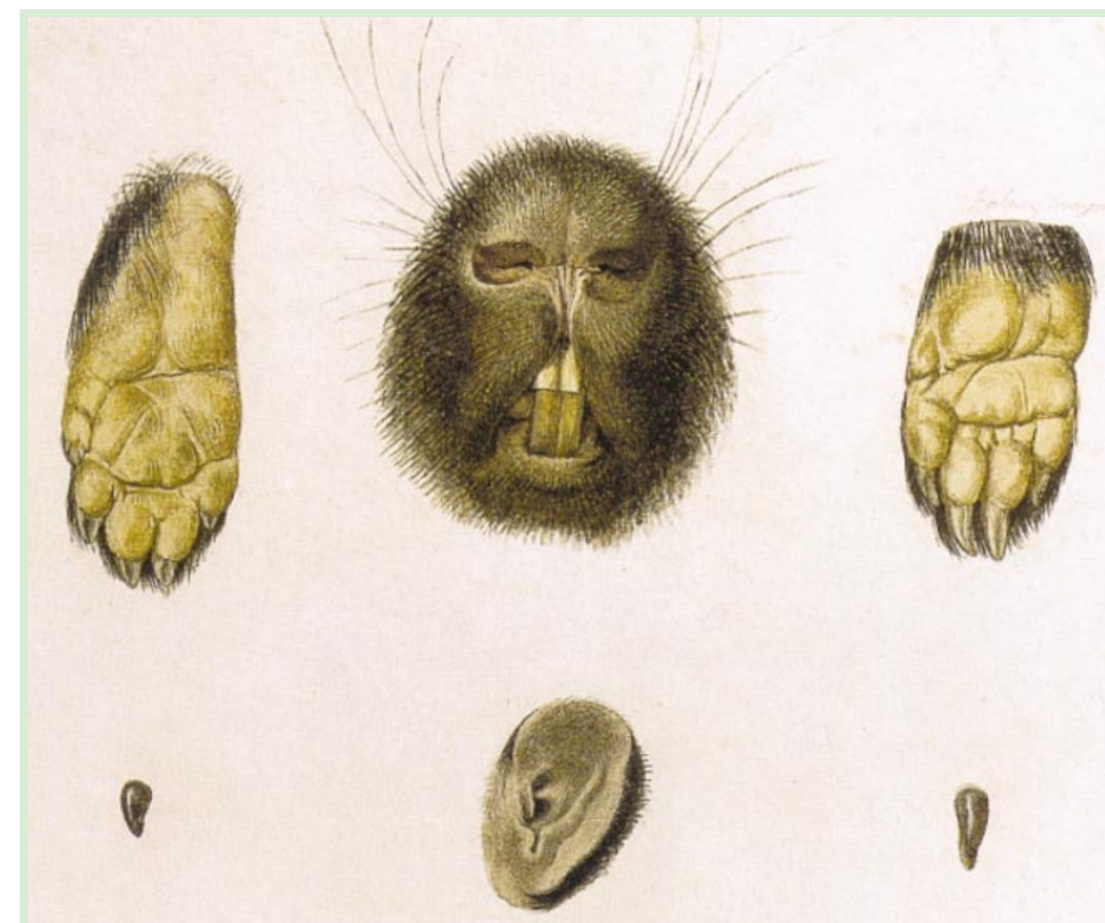

\title{
Through the eye of the lynx
}

Italy is brimming with art treasures, but now and again new troves emerge from obscurity. In The Eye of the Lynx (University of Chicago Press, \$50), art historian David Freedberg brings together a wealth of mostly unknown naturalhistory drawings produced by the world's first modern scientific academy, the Accademia Nazionale dei Lincei (Academy of the Lynxes), which was founded 400 years ago. Revolutionary at the time, the drawings were aided by the use of a new instrument - the microscope - which was created by turning around Galileo's new telescope. Pictured here is a selection of Vincenzo Leonardi's carefully observed details of the anatomy of the common porcupine, drawn in the early seventeenth century. 
From start to finish, this book is so unremittingly silly that it occurred to me that Evans set out to write a sort of postmodernism-inspired spoof, a demonstration that poppycock can get published and passed off as science. I expect that most medical scientists will dismiss the book out of hand. For those who want an informative, scholarly and comprehensive view, I recommend The Placebo Effect: An Interdisciplinary Exploration, edited by Anne Harrington (Harvard University Press, 1997).

The author does not tell us the intended audience for his book, but he supplies definitions of common medical terms and explanations of medical concepts, so I suspect that he is writing for a general audience. Many educated general readers will recognize his empty theorizing, circular reasoning and mis-statements for what they are. But there has always been a ready market for ideas that in a single stroke explain the ills that afflict us and reveal the pathway to health. In modern times these notions often come with the trappings of science and a whiff of scientific plausibility. Some will find in Evans a kindred spirit, and feel their hearts warmed by the knowledge that even something as seemingly elusive as the placebo response can be easily explained.

Walter A. Brown is in the Department of Psychiatry and Human Behaviour, Brown Medical School, and Tufts University School of Medicine, and is at 108 Driftwood Drive, Tiverton, Rhode Island 02878, USA.

\section{First among atoms}

\section{Hydrogen: The Essential Element}

by John S. Rigden

Harvard University Press: 2002. 288 pp.

$\$ 28, £ 19.50$, E28

\section{Daniel Kleppner}

It would be hard to find a better icon for reductionism than the hydrogen atom. It has been said, as John Rigden recalls in Hydrogen: "To understand hydrogen is to understand all of nature." Here he provides abundant evidence to support this extravagant statement. The atom that played a seminal role in the creation of key theories by Niels Bohr, Werner Heisenberg, Erwin Schrödinger and Paul Dirac, not to mention in relativistic quantum electrodynamics, surely occupies a special place in the history of science. Hydrogen makes up most of the known matter in the Universe, and its radiation has told us a great deal about our galaxy and the cosmos. And hydrogen has played principal roles in magnetic resonance and atomic clocks. Such an atom demands to have its story told, and here
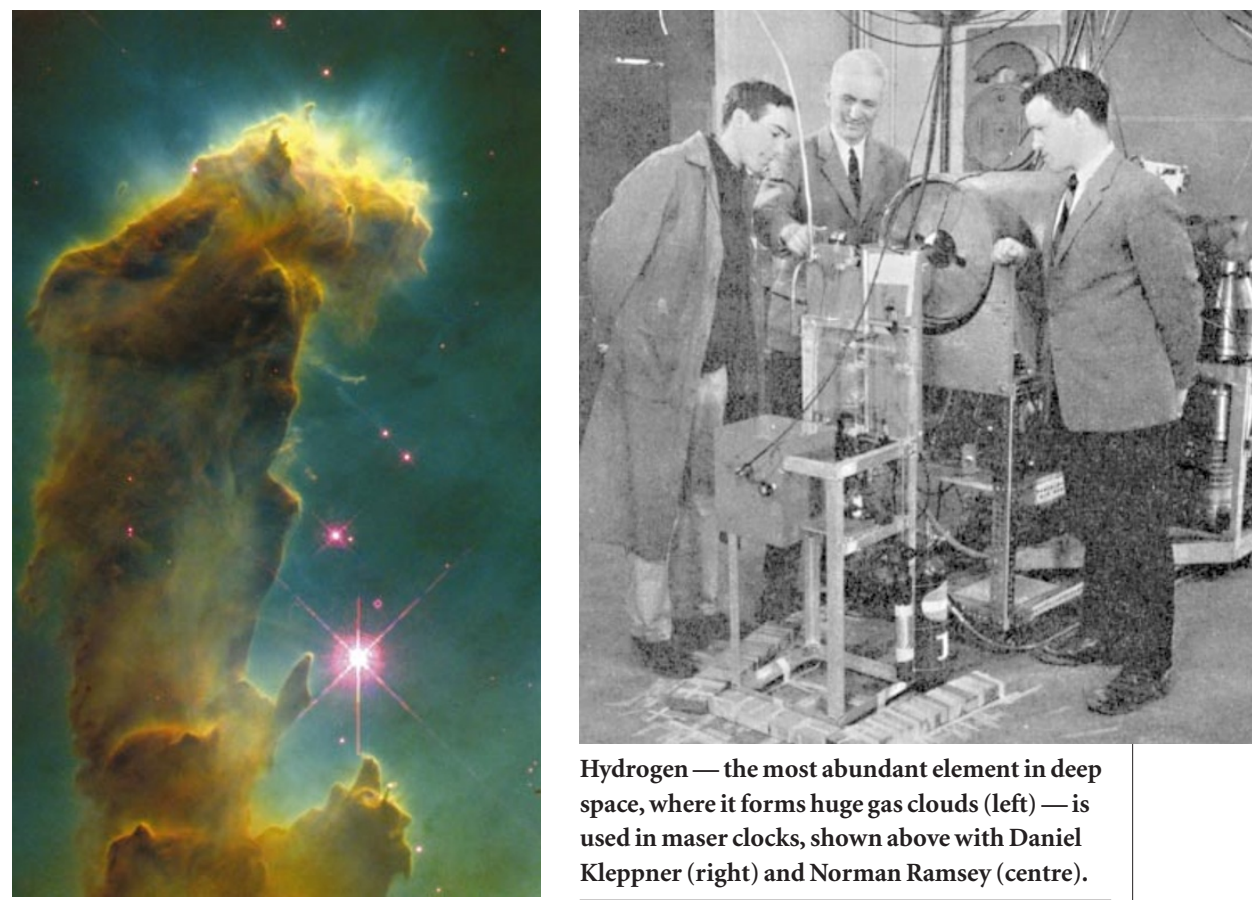

Hydrogen - the most abundant element in deep space, where it forms huge gas clouds (left) - is used in maser clocks, shown above with Daniel Kleppner (right) and Norman Ramsey (centre).

Rigden does an admirable job of telling it.

Rigden narrates the events surrounding discoveries that are generally glossed over in the classroom. He describes how a pythagorean obsession led Johann J. Balmer to discover his prescient empirical formula for hydrogen's wavelengths, and how the finestructure constant emerged from a failed theory - Arnold Sommerfeld's attempt to amalgamate the Bohr model and relativity. This was one of the great failures in physics, and seems to contradict Einstein's dictum that God is subtle but not malicious.

Hydrogen features strongly in Rigden's account of events at Columbia University in New York during the golden years of Isidor I. Rabi from 1930 to 1950 . Rabi attempted to measure the magnetic moment of the proton by improving the molecular-beam method he had brought to the United States from Otto Stern's laboratory in Hamburg. In 1937 this effort culminated in his invention of molecular-beam magnetic resonance, which opened up a new world for spectroscopy and inspired developments such as nuclear magnetic resonance.

It is difficult to overestimate the impact of this invention, both direct and indirect. An early experiment led by a young graduate student, Norman F. Ramsey, indicated that the deuteron (a deuterium nucleus) has a quadrupole moment. This first evidence for non-central nuclear forces constituted a great advance in nuclear physics.

Another experiment revealed that the hyperfine structure of hydrogen disagrees with the value predicted by the Dirac theory. Resolving this discrepancy inspired Julian Schwinger to formulate his version of quantum electrodynamics. Willis Lamb's microwave measurement of the Lamb shift, made in a nearby laboratory, provided further inescapable evidence that the Dirac theory was deficient. And Rabi's magneticresonance methods contributed to the by Edward M. Purcell and Felix Bloch, and the maser and laser by Charles H. Townes, Arthur L. Schawlow and others.

Rigden describes how hydrogen maser atomic clocks are used for deep-space navigation, global positioning and in timekeeping laboratories. He narrates the millionfold advance in spectroscopic precision by Theodor W. Hänsch in his monumental studies of hydrogen. This quest inspired Hänsch's invention of the frequency comb, a revolutionary technique for measuring optical frequencies; the impact of this invention is only starting to be felt. Another advance, too recent for this book, is the creation of antihydrogen atoms at CERN, the European laboratory for particle physics near Geneva. The story of hydrogen is by no means finished.

Hydrogen is aimed at both the public and students of physics. It does not strive for detail at the level of Abraham Pais' Inward Bound (Clarendon Press, 1986), nor does it indulge in speculation - for instance, about whether the creed of reductionism is likely to lose prominence in the coming century, causing the icon of the hydrogen atom to dwindle into a mere curiosity. But its friendly size, straightforward style, numerous illustrations and pithy aphorisms all make for engaging reading.

Daniel Kleppner is in the Department of Physics, Massachusetts Institute of Technology, Cambridge, Massachusetts 02139, USA. discovery of nuclear magnetic resonance 